\title{
Increased circulating interleukin-17 levels in preeclampsia
}

Attila Molvarec, MD, $\mathrm{PhD}^{1}$, Ibolya Czegle, $\mathrm{MD}, \mathrm{PhD}^{2}$, János Szijártó, MSc ${ }^{3}$, János Rigó Jr, $\mathrm{MD}, \mathrm{DSc}^{1}$

1 First Department of Obstetrics and Gynecology, Semmelweis University, Budapest, Hungary

${ }^{2}$ Third Department of Internal Medicine, Semmelweis University, Budapest, Hungary

${ }^{3}$ Central Laboratory, National Institute of Psychiatry and Addictions, Budapest, Hungary

Corresponding author: Attila Molvarec, $\mathrm{MD}, \mathrm{PhD}$

Address: Baross utca 27, Budapest, H-1088, Hungary

E-mail address: molvarec@,freemail.hu

Tel.: +36209571636

Fax: +3613176174 


\begin{abstract}
Increasing evidence suggests that an exaggerated maternal systemic inflammatory response, as well as an angiogenic imbalance plays a central role in the pathogenesis of preeclampsia. In this study, we determined circulating levels of interleukin-17 (IL-17) along with those of angiogenic factors in healthy non-pregnant and pregnant women and preeclamptic patients, and examined whether serum IL-17 levels of preeclamptic patients were related to their clinical features and angiogenic factor concentrations. Fifty-nine preeclamptic patients, 60 healthy pregnant women and 56 healthy non-pregnant women were involved in this casecontrol study. Serum levels of IL-17A were measured by high sensitivity ELISA. Serum total soluble fms-like tyrosine kinase-1 (sFlt-1) and biologically active placental growth factor (PIGF) levels were determined by electrochemiluminescence immunoassay. For statistical analyses, non-parametric methods were applied. Serum IL-17 levels were significantly higher in preeclamptic patients than in healthy non-pregnant and pregnant women. We did not find any relationship between serum IL-17 concentrations of preeclamptic patients and their clinical features and serum sFlt-1 and PlGF levels or sFlt-1/PlGF ratios. However, elevated serum IL-17 level and sFlt-1/PIGF ratio was found to be additive for the risk of preeclampsia, as shown by the substantially higher odds ratios of their combination than of either alone. In conclusion, serum IL-17 levels are increased in preeclampsia, which might contribute to the development of the excessive systemic inflammatory response characteristic of the maternal syndrome of the disease. In addition, elevated serum IL-17 level and sFlt-1/P1GF ratio had an additive (joint) effect in the risk of preeclampsia.
\end{abstract}

Keywords: angiogenesis, inflammation, interleukin-17, preeclampsia, pregnancy, Th17 


\section{Introduction}

Preeclampsia, characterized by hypertension and proteinuria developing after the $20^{\text {th }}$ week of gestation in a previously normotensive woman, is a severe complication of human pregnancy with a worldwide incidence of $4.6(2.7-8.2) \%$ (Abalos et al. 2013). It is one of the leading causes of maternal, as well as perinatal morbidity and mortality, even in developed countries. Despite extensive research, the etiology and pathogenesis of preeclampsia are not completely understood. There is an increasing body of evidence that an exaggerated maternal systemic inflammatory response to pregnancy with activation of both the innate and the adaptive arms of the immune system, as well as an imbalance between circulating angiogenic and anti-angiogenic factors, plays a central role in the pathogenesis of the disease (Redman et al. 1999, Maynard et al. 2003, Saito et al. 2007, Molvarec et al. 2010a, Molvarec et al. 2010b, Szarka et al. 2010).

There are six members of the interleukin (IL)-17-family of cytokines (IL-17A, IL-17B, IL-17C, IL-17D, IL-17E (IL-25) and IL-17F), while the IL-17 receptor family comprises five receptor subunits (IL-17RA, IL-17RB/IL-25R, IL-17RC, IL-17RD/SEF and IL-17RE) (Gaffen 2009). Of them, IL-17A (referred to as IL-17 in the rest of this paper), also known as cytotoxic T lymphocyte-associated antigen 8 (CTLA-8) in rodents, has been most extensively studied. IL-17 is produced by helper and cytotoxic T cells, macrophages, dendritic cells, natural killer, natural killer T, lymphoid tissue inducer cells and $\gamma \delta$ T cells (Onishi and Gaffen 2010). IL-17-producing CD4 cells have been described as a unique subset of helper T cells, which develop via a lineage that is distinct from the T helper type 1 (Th1) and 2 (Th2) lineages (Harrington et al. 2005, Park et al. 2005). Th17 cells are essential for host defence against microbial pathogens, particularly extracellular bacteria and fungi, some protozoa and viruses (O'Quinn et al. 2008).

An important feature of systemic inflammation in preeclampsia is the absence of $\mathrm{Th} 2$ shift characteristic for physiological pregnancy, and thus the predominance of Th1-type immunity (Saito et al. 1999a, Saito et al. 1999b). In addition to the imbalance of Th1 and Th2 cells, alterations in the prevalence of Th17 and regulatory T cells may also contribute to the development of systemic inflammation in preeclampsia (Santner-Nanan et al. 2009, Darmochwal-Kolarz et al. 2012). Our research group has formerly demonstrated that not only the prevalence of Th17 cells, but also that of IL-17-producing CD8 (Tc17) and NK cells is increased in preeclampsia (Toldi et al. 2011).

In the present study, we extended our previous observation and determined serum IL17 levels in a large number of healthy non-pregnant and pregnant women and preeclamptic 
patients. We also measured circulating levels angiogenic factors, and examined whether serum IL-17 levels of preeclamptic patients were related to their clinical features and angiogenic factor concentrations.

\section{Materials and methods}

\subsection{Study patients}

Our study was designed as a case-control study. Fifty-nine preeclamptic patients, 60 healthy pregnant women with uncomplicated pregnancies and 56 healthy non-pregnant women were involved in the study. The study participants were enrolled in the First Department of Obstetrics and Gynecology and in the Department of Obstetrics and Gynecology of Kútvölgyi Clinical Center, at the Semmelweis University, Budapest, Hungary. All women were Caucasian and resided in the same geographic area in Hungary. Exclusion criteria were multifetal gestation, chronic hypertension, diabetes mellitus, autoimmune disease, angiopathy, renal disorder, maternal or fetal infection and fetal congenital anomaly. None of the pregnant women were in active labor, and none had rupture of amniotic membranes. The healthy non-pregnant women were in the early follicular phase of their menstrual cycle (between cycle days 3 and 5), and none of them received hormonal contraception.

Preeclampsia was defined by increased blood pressure $(\geq 140 \mathrm{mmHg}$ systolic or $\geq 90$ $\mathrm{mmHg}$ diastolic on $\geq 2$ occasions at least 6 hours apart) that occurred after the $20^{\text {th }}$ week of gestation in a woman with previously normal blood pressure, accompanied by proteinuria ( $\geq 0.3 \mathrm{~g} / 24 \mathrm{~h}$ or $\geq 1+$ on dipstick in the absence of urinary tract infection). Blood pressure returned to normal by the $12^{\text {th }}$ postpartum week in each preeclamptic study patient. Preeclampsia was regarded as severe if any of the following criteria was present: blood pressure $\geq 160 \mathrm{mmHg}$ systolic or $\geq 110 \mathrm{mmHg}$ diastolic, or proteinuria $\geq 5 \mathrm{~g} / 24 \mathrm{~h}$ (or $\geq 3+$ on dipstick). Pregnant women with eclampsia or HELLP syndrome (hemolysis, elevated liver enzymes, and low platelet count) were not enrolled in this study. Intrauterine growth restriction (IUGR) was diagnosed if the fetal birth weight was below the $10^{\text {th }}$ percentile for gestational age and gender, based on Hungarian birth weight percentiles.

The study protocol was approved by the Regional and Institutional Committee of Science and Research Ethics of the Semmelweis University, and written informed consent was obtained from each patient. The study was conducted in accordance with the Declaration of Helsinki. 


\subsection{Laboratory methods}

Fasting blood samples were taken from an antecubital vein into plain tubes, and then centrifuged at room temperature with a relative centrifugal force of $3000 \mathrm{~g}$ for 10 minutes. The aliquots of serum were stored at $-80{ }^{\circ} \mathrm{C}$ until the analyses. Serum levels of interleukin-17 (IL-17A) were measured by high sensitivity ELISA (eBioscience, San Diego, CA, USA, Cat. No. BMS2017HS) according to the manufacturer's instructions. Serum total soluble fms-like tyrosine kinase-1 (sFlt-1) and biologically active placental growth factor (PlGF) levels were determined by electrochemiluminescence immunoassay (Elecsys, Roche, Mannheim, Germany, Cat. No. 05109523 and 05144671, respectively) on a Cobas e 411 analyzer (Roche, Mannheim, Germany).

\subsection{Statistical analysis}

The normality of continuous variables was assessed using the Shapiro-Wilk's $W$-test. As the continuous variables were not normally distributed, non-parametric statistical methods were used. To compare continuous variables between two groups, the Mann-Whitney $U$-test was applied, whereas to compare them among multiple groups, the Kruskal-Wallis analysis of variance by ranks test was performed. Multiple comparisons of mean ranks for all groups were carried out as post-hoc tests. The Fisher exact and Pearson $\chi^{2}$ tests were used to compare categorical variables between groups. Analysis of covariance (ANCOVA) and multivariate logistic regression were undertaken with adjustment for BMI at blood draw.

Statistical analyses were carried out using the following software: STATISTICA (version 12; StatSoft, Inc., Tulsa, Oklahoma, USA) and Statistical Package for the Social Sciences (version 22 for Windows; SPSS, Inc., Chicago, Illinois, USA). For all statistical analyses, $\mathrm{p}<0.05$ was considered statistically significant.

In the article, data are reported as median (interquartile range) for continuous variables and as number (percentage) for categorical variables.

\section{Results}

\subsection{Patient characteristics}

The clinical characteristics of the study participants are described in Table 1. There was no statistically significant difference in terms of age among the three study groups. Furthermore, no significant differences were observed in gestational age at blood collection 
and the percentage of primiparas between preeclamptic patients and healthy pregnant women. However, all of the other clinical features presented in Table 1 differed significantly among our study groups. Fetal growth restriction was absent in healthy pregnant women, whereas the frequency of this condition was $18.6 \%$ in the preeclamptic group. Twenty-one women $(35.6 \%)$ had severe preeclampsia and 30 patients $(50.8 \%)$ experienced preterm onset $(<37$ weeks) of the disease.

\subsection{Laboratory parameters}

The laboratory parameters of the study subjects are displayed in Table 2. Serum IL-17 levels were significantly higher in preeclamptic patients than in healthy non-pregnant and pregnant women. In addition, the proportion of women with measurable IL-17 concentration was significantly higher in the preeclamptic group compared to the groups of healthy nonpregnant and pregnant women. The differences in serum IL-17 levels between preeclamptic patients and healthy non-pregnant and pregnant women remained significant even after adjustment for BMI at blood draw in ANCOVA (data not shown). Serum levels of sFlt-1 and PlGF, as well as sFlt-1/PlGF ratio, were significantly higher in healthy pregnant than nonpregnant women. Preeclamptic patients had significantly higher sFlt-1 levels and sFlt-1/P1GF ratio and significantly lower PlGF concentrations as compared to healthy pregnant women. Moreover, their sFlt-1 and PlGF levels and sFlt-1/PIGF ratio were significantly higher than those of healthy non-pregnant women.

In the group of preeclamptic patients, no statistically significant differences were found in serum IL-17 concentrations between patients with mild and severe preeclampsia, between patients with preterm and term onset of the disease, or between preeclamptic patients with and without fetal growth restriction (data not shown). However, severely preeclamptic patients had significantly higher sFlt-1 levels compared to those with mild preeclampsia (median (25-75 percentile): 10269 (5267-15420) versus 6113 (3697-8481) pg/ml, p<0.05). In addition, preeclamptic patients with fetal growth restriction or onset of the disease before term had significantly lower PIGF concentrations than those without IUGR (median (25-75 percentile): 70.1 (37.6-104) versus $98.9(68.0-146) \mathrm{pg} / \mathrm{ml}, \mathrm{p}<0.05)$ or with a disease onset at term (median (25-75 percentile): 73.3 (41.9-132) versus $116(91.8-176) \mathrm{pg} / \mathrm{ml}, \mathrm{p}<0.05)$. 


\subsection{Relationship of serum IL-17 levels of preeclamptic patients to their clinical characteristics and laboratory parameters}

We also examined whether serum IL-17 levels of preeclamptic patients were related to their clinical features and angiogenic factor concentrations by dichotomising IL-17 levels based on the median value of the preeclamptic group $(>0.47 \mathrm{pg} / \mathrm{ml}$ or $\leq 0.47 \mathrm{pg} / \mathrm{ml})$. However, we did not find any relationship between serum IL-17 concentrations of preeclamptic patients and their clinical features and serum sFlt-1 and P1GF levels or sFlt-1/P1GF ratios.

Using the Receiver Operating Characteristic (ROC) curve analysis, we determined cutoff values for serum IL-17 concentration ( $>0 \mathrm{pg} / \mathrm{ml}$, sensitivity: $54.2 \%$, specificity: $95.0 \%$; area under curve (AUC) with 95\% CI: $0.74(0.65-0.81))$ and sFlt-1/P1GF ratio (>31.2, sensitivity: 75.9\%, specificity: 74.1\%; AUC (95\% CI): $0.81(0.73-0.88)$ ) to discriminate preeclamptic patients from healthy pregnant women. In a multiple logistic regression model involving both variables, measurable serum IL-17 level and elevated sFlt-1/P1GF ratio were found to be independent predictors of preeclampsia (odds ratios with $95 \%$ confidence intervals: 23.2 (5.63-95.6) and 9.39 (3.29-26.8), respectively, $\mathrm{p}<0.0001$ for both; after adjustment for BMI at blood draw: 31.7 (5.01-200) and 16.8 (3.74-75.9), respectively, $\mathrm{p}<0.001$ for both). Women with measurable serum IL-17 level and elevated sFlt-1/P1GF ratio had substantially higher odds for having preeclampsia than those with elevated sFlt-1/P1GF ratio or measurable serum IL-17 concentration alone (OR (95\% CI): 286 (15.4-5307), $\mathrm{p}<0.0001$ versus 7.31 (2.45-21.8), $\mathrm{p}<0.001$ and 13.0 (2.54-66.4), $\mathrm{p}<0.05$, respectively).

\section{Discussion}

In this study, we measured circulating levels of IL-17 along with those of sFlt-1 and PlGF in a large number of healthy non-pregnant and pregnant women and preeclamptic patients. According to our findings, serum IL-17 levels are significantly higher in preeclamptic patients than in healthy non-pregnant and pregnant women. Serum IL-17 levels of preeclamptic patients were not related to their clinical features and angiogenic factor concentrations. However, the combination of elevated serum IL-17 level and sFlt-1/PlGF ratio was found to be additive for the risk of preeclampsia.

Previous data on circulating IL-17 levels in preeclampsia are controversial. In a study, measuring IL-17 levels in serum samples of 13 women with preeclampsia and 14 normal pregnant women with multiplex bead array, no significant difference was observed (Jonsson et al. 2006). It is noteworthy that the majority of IL-17 serum values in this study were below the detection limit of the assay. Multiplex bead array technology has a high dynamic range, 
and it may not be optimal for evaluating analytes with very low concentration. In a recent study, IL-17 levels were determined in plasma samples of 40 preeclamptic and 40 normotensive pregnant women with ELISA (Ozkan et al. 2014). The authors found lower IL17 levels in the preeclamptic group. However, gestational age in their control group was significantly higher than that of the preeclamptic group. As circulating IL-17 levels have been reported to increase with advancing gestation (Martinez-Garcia et al. 2011), the difference in gestational age might confound their results. In our study, serum IL-17 levels were evaluated in a larger number of preeclamptic patients and healthy pregnant women (matched for age and gestational age) with a high sensitivity ELISA. In addition, we also involved healthy nonpregnant women in our study. Our results are consistent with previous studies demonstrating higher prevalence of peripheral blood Th17 cells and increased expression of RORc mRNA in peripheral blood mononuclear cells and in the decidua in preeclamptic patients (SantnerNanan et al. 2009, Jianjun et al. 2010, Toldi et al. 2011, Darmochwal-Kolarz et al. 2012).

Increased circulating IL-17 levels observed in preeclampsia might contribute to the development of generalized intravascular inflammatory reaction characteristic of the maternal syndrome of the disease. IL-17 induces pro-inflammatory responses by triggering the expression of other cytokines (IL-6, granulocyte colony-stimulating factor, tumor necrosis factor- $\alpha$ ), chemokines (CXCL1, CXCL2, CCL20), inflammatory effectors (acute phase proteins, complement) and antimicrobial proteins (defensins, mucins) (Onishi and Gaffen 2010). Indeed, preeclampsia has already been demonstrated to be associated with elevated amounts of pro-inflammatory cytokines, chemokines, acute phase proteins and complement activation products in the maternal circulation (Molvarec et al. 2009a, Derzsy et al. 2010, Szarka et al. 2010). Furthermore, IL-17 has been linked to the pathogenesis of several autoimmune and inflammatory disorders, such as rheumatoid arthritis, systemic lupus erythematosus, inflammatory bowel disease, psoriasis and multiple sclerosis (Onishi and Gaffen 2010).

In a rat model, IL-17 has been shown to induce circulating Th17 cells, placental oxidative stress, the production of autoantibodies activating the angiotensin II type 1 receptor (AT1-AA) and hypertension during pregnancy (Dhillion et al. 2012). Additionally, administration of IL-17 soluble receptor C (an inhibitor of IL-17 function) suppressed circulating Th17 cells, oxidative stress, AT1-AA and hypertension in the reduced uterine perfusion pressure (RUPP) model of preeclampsia (Cornelius et al. 2013a). Another study of the same research group indicated that endothelin-1 pathway is not a mechanism by which IL17 causes hypertension during pregnancy (Cornelius et al. 2013b). Although we did not find a 
relationship between serum IL-17 levels and blood pressure values in our preeclamptic group, the process responsible for blood pressure elevation in this multifactorial disorder is more complex and may involve the interplay of numerous factors in addition to the effect of IL-17.

Accumulating data indicate the central role of circulating angiogenic factors and their antagonists in the development of preeclampsia (Maynard et al. 2003, Molvarec et al. 2010a, Molvarec et al. 2010b). In this study, we also measured serum sFlt-1 and P1GF concentrations by electrochemiluminescence immunoassay. However, increased sFlt-1 and decreased PlGF levels were not related to serum IL-17 concentrations in women with preeclampsia, suggesting that angiogenic imbalance and alterations in circulating IL-17 levels are independent mechanisms in the pathogenesis of preeclampsia. Instead, elevated serum IL-17 level and sFlt-1/P1GF ratio had an additive (joint) effect in the risk of preeclampsia, as shown by the substantially higher odds ratios of their combination than of either alone.

In our study, the similar circulating IL-17 concentrations of preeclamptic patients regardless of the severity of the disease or the presence of fetal growth restriction might be explained by the multifactorial etiology of preeclampsia. Several genetic, behavioural and environmental factors need to interact to produce the complete picture of this pregnancyspecific disorder. Our research group reported various genetic and soluble factors that were associated with the severity or complications of preeclampsia (Molvarec et al. 2007, Molvarec et al. 2008, Molvarec et al. 2009b, Rosta et al. 2009).

There is an increasing body of evidence for distinct preterm and term phenotypes of preeclampsia with differences in maternal and fetal characteristics and pathogenetic features (Vatten and Skjaerven 2004, Phillips et al. 2010). In our study, no significant differences were observed in serum IL-17 concentrations between preeclamptic patients with preterm and term onset of the disease. We hypothesize that the development of these two different disease entities converges at maternal systemic inflammation with elevated amounts of IL-17 in the maternal circulation. Nevertheless, further studies are required with involvement of a larger number of patients with preterm and especially early-onset preeclampsia (disease onset $<34$ weeks) to support our hypothesis.

In conclusion, according to our findings, serum IL-17 levels are increased in women with preeclampsia, which might contribute to the development of the excessive systemic inflammatory response characteristic of the maternal syndrome of the disease. In addition, elevated serum IL-17 level and sFlt-1/P1GF ratio had an additive (joint) effect in the risk of preeclampsia. 


\section{Acknowledgements}

This work was supported by the János Bolyai Research Scholarship of the Hungarian Academy of Sciences, as well as by a research grant from the Hungarian Scientific Research Fund (PD 109094).

\section{References}

Abalos, E., Cuesta, C., Grosso, A. L., Chou, D. and Say, L. (2013). Global and regional estimates of preeclampsia and eclampsia: a systematic review. Eur J Obstet Gynecol Reprod Biol 170, 1-7.

Cornelius, D. C., Hogg, J. P., Scott, J., Wallace, K., Herse, F., Moseley, J., et al. (2013a). Administration of interleukin-17 soluble receptor C suppresses TH17 cells, oxidative stress, and hypertension in response to placental ischemia during pregnancy. Hypertension 62, 1068-1073.

Cornelius, D. C., Wallace, K., Kiprono, L., Dhillon, P., Moseley, J. and LaMarca, B. (2013b). Endothelin-1 is not a Mechanism of IL-17 Induced Hypertension during Pregnancy. Med J Obstet Gynecol 1, 1006.

Darmochwal-Kolarz, D., Kludka-Sternik, M., Tabarkiewicz, J., Kolarz, B., Rolinski, J., Leszczynska-Gorzelak, B., et al. (2012). The predominance of Th17 lymphocytes and decreased number and function of Treg cells in preeclampsia. J Reprod Immunol 93, $75-81$.

Derzsy, Z., Prohaszka, Z., Rigo, J., Jr., Fust, G. and Molvarec, A. (2010). Activation of the complement system in normal pregnancy and preeclampsia. Mol Immunol 47, 1500 1506.

Dhillion, P., Wallace, K., Herse, F., Scott, J., Wallukat, G., Heath, J., et al. (2012). IL-17mediated oxidative stress is an important stimulator of AT1-AA and hypertension during pregnancy. Am J Physiol Regul Integr Comp Physiol 303, R353-358.

Gaffen, S. L. (2009). Structure and signalling in the IL-17 receptor family. Nat Rev Immunol $9,556-567$.

Harrington, L. E., Hatton, R. D., Mangan, P. R., Turner, H., Murphy, T. L., Murphy, K. M., et al. (2005). Interleukin 17-producing CD4+ effector $\mathrm{T}$ cells develop via a lineage distinct from the T helper type 1 and 2 lineages. Nat Immunol 6, 1123-1132. 
Jianjun, Z., Yali, H., Zhiqun, W., Mingming, Z. and Xia, Z. (2010). Imbalance of T-cell transcription factors contributes to the Th1 type immunity predominant in preeclampsia. Am J Reprod Immunol 63, 38-45.

Jonsson, Y., Ruber, M., Matthiesen, L., Berg, G., Nieminen, K., Sharma, S., et al. (2006). Cytokine mapping of sera from women with preeclampsia and normal pregnancies. $\mathrm{J}$ Reprod Immunol 70, 83-91.

Martinez-Garcia, E. A., Chavez-Robles, B., Sanchez-Hernandez, P. E., Nunez-Atahualpa, L., Martin-Maquez, B. T., Munoz-Gomez, A., et al. (2011). IL-17 increased in the third trimester in healthy women with term labor. Am J Reprod Immunol 65, 99-103.

Maynard, S. E., Min, J. Y., Merchan, J., Lim, K. H., Li, J., Mondal, S., et al. (2003). Excess placental soluble fms-like tyrosine kinase 1 (sFlt1) may contribute to endothelial dysfunction, hypertension, and proteinuria in preeclampsia. J Clin Invest 111, 649658.

Molvarec, A., Prohaszka, Z., Nagy, B., Kalabay, L., Szalay, J., Fust, G., et al. (2007). Association of increased serum heat shock protein 70 and $\mathrm{C}$-reactive protein concentrations and decreased serum alpha(2)-HS glycoprotein concentration with the syndrome of hemolysis, elevated liver enzymes, and low platelet count. J Reprod Immunol 73, 172-179.

Molvarec, A., Jermendy, A., Nagy, B., Kovacs, M., Varkonyi, T., Hupuczi, P., et al. (2008). Association between tumor necrosis factor (TNF)-alpha G-308A gene polymorphism and preeclampsia complicated by severe fetal growth restriction. Clin Chim Acta 392, $52-57$.

Molvarec, A., Kalabay, L., Derzsy, Z., Szarka, A., Halmos, A., Stenczer, B., et al. (2009a). Preeclampsia is associated with decreased serum alpha(2)-HS glycoprotein (fetuin-A) concentration. Hypertens Res 32, 665-669.

Molvarec, A., Rigo, J., Jr., Lazar, L., Balogh, K., Mako, V., Cervenak, L., et al. (2009b). Increased serum heat-shock protein 70 levels reflect systemic inflammation, oxidative stress and hepatocellular injury in preeclampsia. Cell Stress Chaperones 14, 151-159.

Molvarec, A., Ito, M., Shima, T., Yoneda, S., Toldi, G., Stenczer, B., et al. (2010a). Decreased proportion of peripheral blood vascular endothelial growth factorexpressing $\mathrm{T}$ and natural killer cells in preeclampsia. Am J Obstet Gynecol 203, 567 e561-568.

Molvarec, A., Szarka, A., Walentin, S., Szucs, E., Nagy, B. and Rigo, J., Jr. (2010b). Circulating angiogenic factors determined by electrochemiluminescence immunoassay 
in relation to the clinical features and laboratory parameters in women with preeclampsia. Hypertens Res 33, 892-898.

O'Quinn, D. B., Palmer, M. T., Lee, Y. K. and Weaver, C. T. (2008). Emergence of the Th17 pathway and its role in host defense. Adv Immunol 99, 115-163.

Onishi, R. M. and Gaffen, S. L. (2010). Interleukin-17 and its target genes: mechanisms of interleukin-17 function in disease. Immunology 129, 311-321.

Ozkan, Z. S., Simsek, M., Ilhan, F., Deveci, D., Godekmerdan, A. and Sapmaz, E. (2014). Plasma IL-17, IL-35, interferon-gamma, SOCS3 and TGF-beta levels in pregnant women with preeclampsia, and their relation with severity of disease. J Matern Fetal Neonatal Med 27, 1513-1517.

Park, H., Li, Z., Yang, X. O., Chang, S. H., Nurieva, R., Wang, Y. H., et al. (2005). A distinct lineage of CD4 T cells regulates tissue inflammation by producing interleukin 17 . Nat Immunol 6, 1133-1141.

Phillips, J. K., Janowiak, M., Badger, G. J. and Bernstein, I. M. (2010). Evidence for distinct preterm and term phenotypes of preeclampsia. J Matern Fetal Neonatal Med 23, 622626.

Redman, C. W., Sacks, G. P. and Sargent, I. L. (1999). Preeclampsia: an excessive maternal inflammatory response to pregnancy. Am J Obstet Gynecol 180, 499-506.

Rosta, K., Molvarec, A., Enzsoly, A., Nagy, B., Ronai, Z., Fekete, A., et al. (2009). Association of extracellular superoxide dismutase (SOD3) Ala40Thr gene polymorphism with pre-eclampsia complicated by severe fetal growth restriction. Eur J Obstet Gynecol Reprod Biol 142, 134-138.

Saito, S., Sakai, M., Sasaki, Y., Tanebe, K., Tsuda, H. and Michimata, T. (1999a). Quantitative analysis of peripheral blood Th0, Th1, Th2 and the Th1:Th2 cell ratio during normal human pregnancy and preeclampsia. Clin Exp Immunol 117, 550-555.

Saito, S., Umekage, H., Sakamoto, Y., Sakai, M., Tanebe, K., Sasaki, Y., et al. (1999b). Increased T-helper-1-type immunity and decreased T-helper-2-type immunity in patients with preeclampsia. Am J Reprod Immunol 41, 297-306.

Saito, S., Shiozaki, A., Nakashima, A., Sakai, M. and Sasaki, Y. (2007). The role of the immune system in preeclampsia. Mol Aspects Med 28, 192-209.

Santner-Nanan, B., Peek, M. J., Khanam, R., Richarts, L., Zhu, E., Fazekas de St Groth, B., et al. (2009). Systemic increase in the ratio between Foxp3+ and IL-17-producing CD4+ $\mathrm{T}$ cells in healthy pregnancy but not in preeclampsia. J Immunol 183, 7023-7030. 
Szarka, A., Rigo, J., Jr., Lazar, L., Beko, G. and Molvarec, A. (2010). Circulating cytokines, chemokines and adhesion molecules in normal pregnancy and preeclampsia determined by multiplex suspension array. BMC Immunol 11, 59.

Toldi, G., Rigo, J., Jr., Stenczer, B., Vasarhelyi, B. and Molvarec, A. (2011). Increased prevalence of IL-17-producing peripheral blood lymphocytes in pre-eclampsia. Am J Reprod Immunol 66, 223-229.

Vatten, L. J. and Skjaerven, R. (2004). Is pre-eclampsia more than one disease? BJOG 111, 298-302. 
Table 1. Clinical characteristics of healthy non-pregnant and pregnant women and preeclamptic patients

\begin{tabular}{|l|l|l|l|}
\hline & $\begin{array}{l}\text { Healthy non- } \\
\text { pregnant women } \\
(\mathrm{n}=56)\end{array}$ & $\begin{array}{l}\text { Healthy pregnant } \\
\text { women }(\mathrm{n}=60)\end{array}$ & $\begin{array}{l}\text { Preeclamptic patients } \\
(\mathrm{n}=59)\end{array}$ \\
\hline Age (years) & $28(23-34)$ & $30(28-32)$ & $29(26-32)$ \\
\hline BMI at blood draw $\left(\mathrm{kg} / \mathrm{m}^{2}\right)$ & $20.8(19.6-23.0)$ & $25.8(24.3-27.9)^{\mathrm{b}}$ & $29.8(26.9-33.3)^{\mathrm{b}, \mathrm{d}}$ \\
\hline Smokers & $13(23.2 \%)$ & $0(0 \%)^{\mathrm{b}}$ & $3(5.1 \%)^{\mathrm{a}}$ \\
\hline Primiparas & n.a. & $37(61.7 \%)$ & $38(64.4 \%)$ \\
\hline $\begin{array}{l}\text { Systolic blood pressure at blood } \\
\text { draw (mmHg) }\end{array}$ & $110(110-120)$ & $110(107-120)$ & $164(156-180)^{\mathrm{b}, \mathrm{d}}$ \\
\hline $\begin{array}{l}\text { Diastolic blood pressure at blood } \\
\text { draw (mmHg) }\end{array}$ & $80(70-80)$ & $70(60-80)^{\mathrm{b}}$ & $100(97-110)^{\mathrm{b}, \mathrm{d}}$ \\
\hline $\begin{array}{l}\text { Gestational age at blood draw } \\
\text { (weeks) }\end{array}$ & n.a. & $36(36-37)$ & $37(36-39)$ \\
\hline $\begin{array}{l}\text { Gestational age at delivery } \\
\text { (weeks) }\end{array}$ & n.a. & $39(38-40)$ & $38(37-39)^{\mathrm{d}}$ \\
\hline Fetal birth weight (grams) & n.a. & $3450(3150-3700)$ & $3150(2450-3500)^{\mathrm{d}}$ \\
\hline Fetal growth restriction & n.a. & $0(0 \%)$ & $11(18.6 \%)^{\mathrm{d}}$ \\
\hline
\end{tabular}

Data are presented as median (interquartile range) for continuous variables and as number (percentage) for categorical variables

n.a.: not applicable; BMI: body mass index

${ }^{\mathrm{a}} \mathrm{p}<0.05$ versus healthy non-pregnant women

${ }^{\mathrm{b}} \mathrm{p}<0.001$ versus healthy non-pregnant women

${ }^{c} p<0.05$ preeclamptic patients versus healthy pregnant women

${ }^{d} p<0.001$ preeclamptic patients versus healthy pregnant women 
Table 2. Laboratory parameters of healthy non-pregnant and pregnant women and preeclamptic patients

\begin{tabular}{|l|l|l|l|}
\hline & $\begin{array}{l}\text { Healthy non- } \\
\text { pregnant women } \\
(\mathrm{n}=56)\end{array}$ & $\begin{array}{l}\text { Healthy pregnant } \\
\text { women }(\mathrm{n}=60)\end{array}$ & $\begin{array}{l}\text { Preeclamptic patients } \\
(\mathrm{n}=59)\end{array}$ \\
\hline Serum IL-17 level (pg/ml) & $0(0-0)$ & $0(0-0)$ & $0.47(0-0.53)^{\mathrm{a}, \mathrm{b}}$ \\
\hline Measurable serum IL-17 level & $5(8.9 \%)$ & $3(5.0 \%)$ & $32(54.2 \%)^{\mathrm{a}, \mathrm{b}}$ \\
\hline Serum sFlt-1 level $(\mathrm{pg} / \mathrm{ml})$ & $76.3(67.1-83.6)^{*}$ & $3252(2509-4751)^{\dagger, \mathrm{a}}$ & $6814(3736-12720)^{\ddagger, \mathrm{a}, \mathrm{b}}$ \\
\hline Serum PlGF level $(\mathrm{pg} / \mathrm{ml})$ & $16.2(14.0-18.0)^{*}$ & $183(126-307)^{\dagger, \mathrm{a}}$ & $98.0(63.7-146)^{\ddagger}$ \\
\hline Serum sFlt-1/PlGF ratio & $4.79(3.82-5.52)^{*}$ & $15.6(8.52-36.6)^{\dagger, \mathrm{a}}$ & $70.5(31.8-144)^{\sharp}, \mathrm{a,b}$ \\
\hline
\end{tabular}

Data are presented as median (interquartile range) for continuous variables and as number (percentage) for categorical variables

IL-17: interleukin-17; sFlt-1: soluble fms-like tyrosine kinase-1; P1GF: placental growth factor

${ }^{*} \mathrm{n}=52{ }^{\dagger} \mathrm{n}=58 \quad{ }^{\ddagger} \mathrm{n}=54$

${ }^{\mathrm{a}} \mathrm{p}<0.001$ versus healthy non-pregnant women

${ }^{\mathrm{b}} \mathrm{p}<0.001$ preeclamptic patients versus healthy pregnant women 